Acta Crystallographica Section B

Structural

Science

ISSN 0108-7681

Editor: Carolyn P. Brock

\title{
A reinterpretation of the phase transitions in $\mathrm{Na}_{2} \mathrm{CO}_{3}$ Alla Arakcheeva and Gervais Chapuis
}

Copyright $(\odot$ International Union of Crystallography

Author(s) of this paper may load this reprint on their own web site provided that this cover page is retained. Republication of this article or its storage in electronic databases or the like is not permitted without prior permission in writing from the IUCr. 
Acta Crystallographica Section B

Structural

Science

ISSN 0108-7681

\section{Alla Arakcheeva* and Gervais Chapuis}

Ecole Polytechnique Fédérale de Lausanne, Institut de Physique de la Matière Complexe, BSP, 1015 Lausanne, Switzerland

Correspondence e-mail:

Alla.Arakcheeva@epfl.ch

\section{A reinterpretation of the phase transitions in $\mathrm{Na}_{2} \mathrm{CO}_{3}$}

Based on the structural data of phases $\alpha$ (hexagonal; 756$972 \mathrm{~K}$ ), $\beta$ (monoclinic; 605-751 K), $\gamma$ (incommensurate, monoclinic; $295 \mathrm{~K}$ ) and $\delta$ (lock-in, monoclinic; $110 \mathrm{~K}$ ) of sodium carbonate, $\mathrm{Na}_{2} \mathrm{CO}_{3}$, we could draw a parallel between the phase transitions and the evolution of the second coordination sphere of the $\mathrm{C}$ atoms. The temperaturedependent structures observed in the $\beta$ phase are reproduced in the incommensurate $\gamma$ phase as a modulation wave, which relates to the content of the symmetrically equivalent $\{110\}$ lattice planes in the $\alpha$ phase. By decreasing the temperature, the phase transitions are associated with a stepwise increase in the number of $\mathrm{Na}$ ions participating in the second coordination sphere of the $\mathrm{C}$ atoms. Over the full temperature range, this number increases from 3 to 7 . The $\mathrm{C}-\mathrm{O}$ distances and the mobility of the $\mathrm{O}$ atoms depends on the number of $\mathrm{Na}$ ions in the vicinity of the $\mathrm{C}$ atoms.

\section{Introduction}

Incommensurate structures are conveniently described in the superspace formalism. The obvious advantage is that their structures can recover lattice periodicity, in a space varying between four and six dimensions. If the incommensurate structure is part of a sequence of phases where both commensurate and incommensurate structures can exist, it appears that the theoretical tools are still incomplete in order to give a coherent view of the complete set of phases occurring at various temperatures or pressures. From the theoretical considerations of Parlinski \& Chapuis (1993), the fundamental role of the atomic interactions of the second coordination sphere (cation-cation for the anion containing compounds) was shown for the 'commensurate-incommensurate' phase transition. Based on the sequences of the temperaturedependent phases of $\mathrm{Na}_{2} \mathrm{CO}_{3}$ (Fig. 1), we would like to illustrate this theoretical prediction and show that the specific cation-cation interactions can be associated with each of the phase transitions which have been identified. In this context, the interaction must be understood in terms of the number of second nearest-neighbour interactions which are characterized by distances smaller than a limiting distance which will be discussed later.

The temperature-dependent phase transitions of $\mathrm{Na}_{2} \mathrm{CO}_{3}$ have been widely studied (Wolff \& Tuinstra, 1986; Harris \& Salje, 1992; Swainson et al., 1995; Harris \& Dove, 1995; Harris et al., 1996; Dušek et al., 2003). The following sequence of phases is supported by precise structural investigations which have been repeatedly confirmed:

(i) $\alpha: T=\sim 754-1000 \mathrm{~K}$; space group $P 6_{3} / m m c$; 10 refinements were reported by Swainson et al. (1995) for different temperatures; unit-cell parameters: $a=5.209$ (2) $\AA$ and $c$
Received 22 November 2004 Accepted 14 October 2005 
varies from 6.454 to $6.676 \AA$ [the values $a_{M}=9.022$ (4) and $b_{M}=5.209 \pm 0.002 \AA$ refer to the monoclinic setting].

(ii) $\beta: T=605-754 \mathrm{~K}$; space group $C 2 / m$; about 20 refinements were performed by Swainson et al. (1995) for different temperatures; unit-cell parameters: $a=9.00$ (2), $b=5.23$ (2) Å, the $c$ parameter and monoclinic angle $\beta$ vary from 6.21 to $6.44 \AA$ and from 90 to $99.33^{\circ}$, respectively.

(iii) $\gamma$ (incommensurate): $T=170-605 \mathrm{~K}$; super-space group $C 2 / m(\alpha 0 \gamma) 0 s$; the study was performed at $295 \mathrm{~K}$ (Dušek et al., 2003); unit-cell parameters $a=8.920$ (7), $b=5.245$ (5), $c=$ 6.050 (5) ̊, $\beta=101.35(8)^{\circ}, \mathbf{q}=$ [0.182 (1), 0, 0.322 (1)].

(iv) $\delta$ (lock-in): $T \leq 170 \mathrm{~K}$; space group $P 2_{1} / n$ [equal to superspace group $\left.C 2 / m\left(\frac{1}{6} 0 \frac{1}{3}\right) 0 s\right]$; the structural data were obtained at $110 \mathrm{~K}$ (Dušek et al., 2003); unit-cell parameters $a=$ 8.898 (7), $b=5.237$ (5), $c=5.996(5) \AA, \beta=101.87(8)^{\circ}, \mathbf{q}=\left[\frac{1}{6}\right.$, $\left.\left.0, \frac{1}{3}\right)\right]$.

In all the available publications, the phase transitions are associated with the tilt of the triangular $\mathrm{CO}_{3}$ rigid units. However, their origins have been explained in two different ways. The $\alpha \rightarrow \beta$ structure transformation was associated with a ferroelastic second-order phase transition by Swainson et al. (1995), with the spontaneous shear strain (namely, the component $\left.\epsilon_{5}=a \cos \beta^{*} / a_{0}\right)$ as the primary order parameter. In this model, the carbonate rotation simply follows rather than drives the shear strain. A lattice dynamics calculation based on a $\mathrm{C}-\mathrm{O}$ and $\mathrm{Na}-\mathrm{O}$ rigid-bond model was invoked to justify this model. In Dušek et al. (2003), the origin of the $\beta \rightarrow \gamma$ phase transition was associated with the tilting of the carbonate groups. The unsaturated bonding potential of the $\mathrm{Na}^{+}$ions was invoked in order to explain the progressive tilt of the $\mathrm{CO}_{3}$ group. Indeed, the bond-valence sums (BVS) calculated by Dušek et al. (2003) demonstrate this tendency for one $\mathrm{Na}^{+}$ion [BVS: $0.71(\alpha) \rightarrow 0.80(\beta) \rightarrow 0.94(\gamma) \rightarrow 1.00(\delta)$ ]. However, the other two $\mathrm{Na}^{+}$ions become over-saturated with $\mathrm{Na}-\mathrm{O}$ bonds [BVS: $1.06(\alpha) \rightarrow 1.25(\beta) \rightarrow 1.35(\gamma) \rightarrow 1.4$ $(\delta)$ ], which is not favorable for the structure stabilization. Hence, the question about the nature of the phase transitions is still open and the origin of the tilt of the $\mathrm{CO}_{3}$ triangle at lower temperature remains without a plausible explanation.

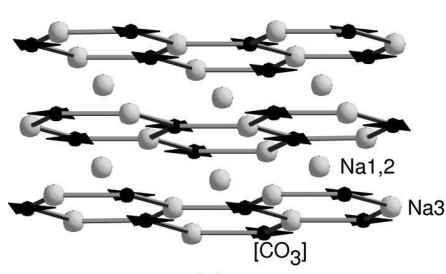

(a)

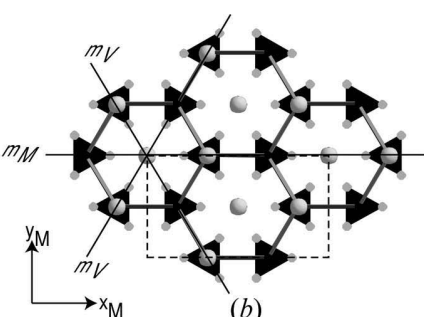

(b)
Figure 1

The crystal structure of $\mathrm{Na}_{2} \mathrm{CO}_{3}:(a)$ perspective view and $(b)$ the $(001)$ projection. The $\mathrm{Na} 3$ and $\left[\mathrm{CO}_{3}\right]$ ions form graphite-like layers, parallel to (001) and stacked in the third direction. The Na1,2 ions are located in the hexagonal channels. The three atomic planes of the $\{110\}_{\text {hex }}$ family contain all the atoms of the structure. They are equivalent in the hexagonal $\alpha$ phase and indicated as $m_{M}$ and $m_{V}$ corresponding to one mirror plane and two 'virtual mirror' planes in the monoclinic modifications. The monoclinic unit cell is shown with a dashed line.
No evidence of any discontinuity at the transition temperatures was mentioned in any of the available publications dedicated to $\mathrm{Na}_{2} \mathrm{CO}_{3}$ crystal structures. Only the $\mathrm{C}-\mathrm{O}$ and $\mathrm{Na}-\mathrm{O}$ interactions were considered in order to justify the transition mechanism.

In the present article, we show that by decreasing the temperature some dramatic changes occur in this structure between non-O atoms, which are associated with the phase transformations. Based on an analysis of the structural data obtained from Swainson et al. (1995) and Dušek et al. (2003) for all modifications mentioned above, we postulate that the gradual strengthening of the $\mathrm{C}-\mathrm{Na}$ interactions (decrease of $\mathrm{C}-\mathrm{Na}$ distances from 3.4 to $3.0 \pm 0.1 \AA$ ) is the main structural feature underlying all the phase transitions observed over decreasing temperatures. All the temperature-dependent

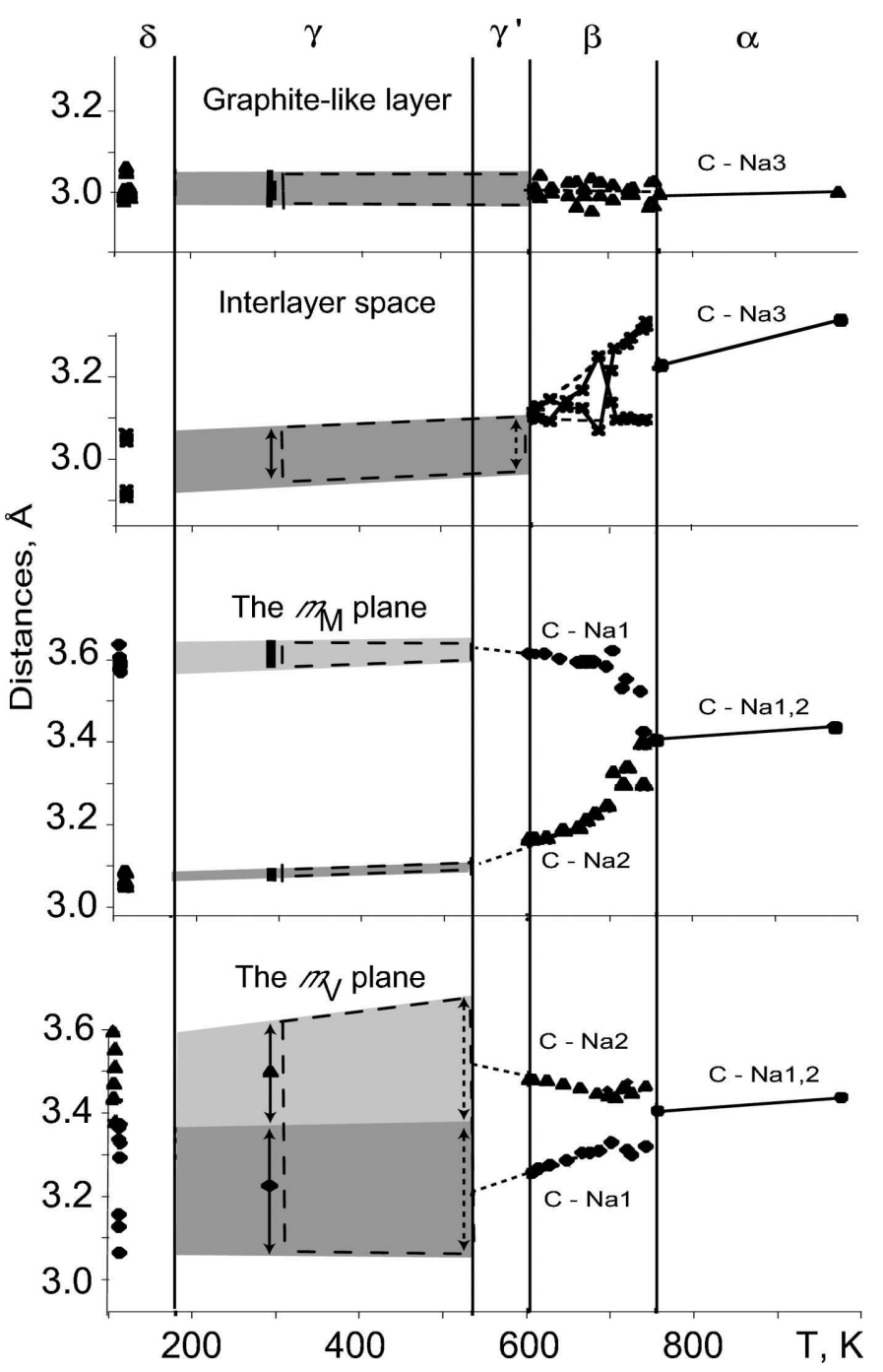

Figure 2

Temperature dependence of the $\mathrm{C}-\mathrm{Na}$ distances in the $110-972 \mathrm{~K}$ range. Various dots and solid lines indicate the experimental data published in Swainson et al. (1995) and Dušek et al. (2003). The solid vertical lines correspond to the phase transition temperatures. The gray areas indicate modulated distances and the double-sided arrows indicate the anti-phase modulations in the two $m_{V}$ planes. The dashed lines are approximations. The incommensurate $\gamma^{\prime}$ phase is derived from a reinterpretation of the experimental data. 
transformations, such as the increase of the monoclinic $\beta$ angle (justifying the $\epsilon_{5}=a \cos \beta * / a_{0}$ shear strain component), the tilt of the $\mathrm{CO}_{3}$ triangles, the difference between the $\mathrm{O} 1$ and $\mathrm{O} 2$ atomic displacements and the $\mathrm{C}-\mathrm{O}$ distances, follow directly from the decrease of the $\mathrm{C}-\mathrm{Na}$ distances on decreasing the temperature. The result is a stepwise formation of $\mathrm{C}-\mathrm{Na}$ bonds, i.e. $\mathrm{C}-\mathrm{Na}$ distances shorter than $3.1 \AA$. [It is worth mentioning that the $\mathrm{C}-\mathrm{Na}$ distances, 2.62-2.92 $\AA$, characteristic of oxygen-free $\mathrm{Na}_{2} \mathrm{C}_{2}$ (Hemmersbach et al., 1999) are close to these values.] We show that each successive phase transition is correlated with the formation of an additional bond in the second coordination sphere of $\mathrm{C}$ atoms. The $\mathrm{C}-$ $\mathrm{Na}$ bond formation is not continuous at the $\alpha \rightarrow \beta$ transition temperature. The $\alpha \rightarrow \beta$ transformation mechanism is more complex than the description given in Swainson et al. (1995). From the experimental data, our analysis allows the prediction and confirmation of the existence of an additional modification of the incommensurate $\gamma$ phase.

\section{The phase transitions and the $\mathrm{C}-\mathrm{Na}$ bond formation}

The structure of $\mathrm{Na}_{2} \mathrm{CO}_{3}$ (Fig. 1) can be described as graphitelike layers formed by $\mathrm{Na} 3$ and $\mathrm{CO}_{3}$ ions, which are stacked along the hexagonal axis. Additional Na1,2 ions are located in the hexagonal channels. The graphite-like layers are regular in all modifications and all the $\mathrm{C}-\mathrm{Na} 3$ bonds are characterized by the minimum temperature-independent distances, $3.00 \pm 0.05 \AA$ (Fig. 2). The two other types of $\mathrm{C}-\mathrm{Na}$ distances found in the hexagonal high-temperature $\alpha$ phase are longer $(\sim 3.4 \AA)$, pointing to weak interactions. One of them corresponds to the $\mathrm{C}-\mathrm{Na1}, 2$ distance between the layer and

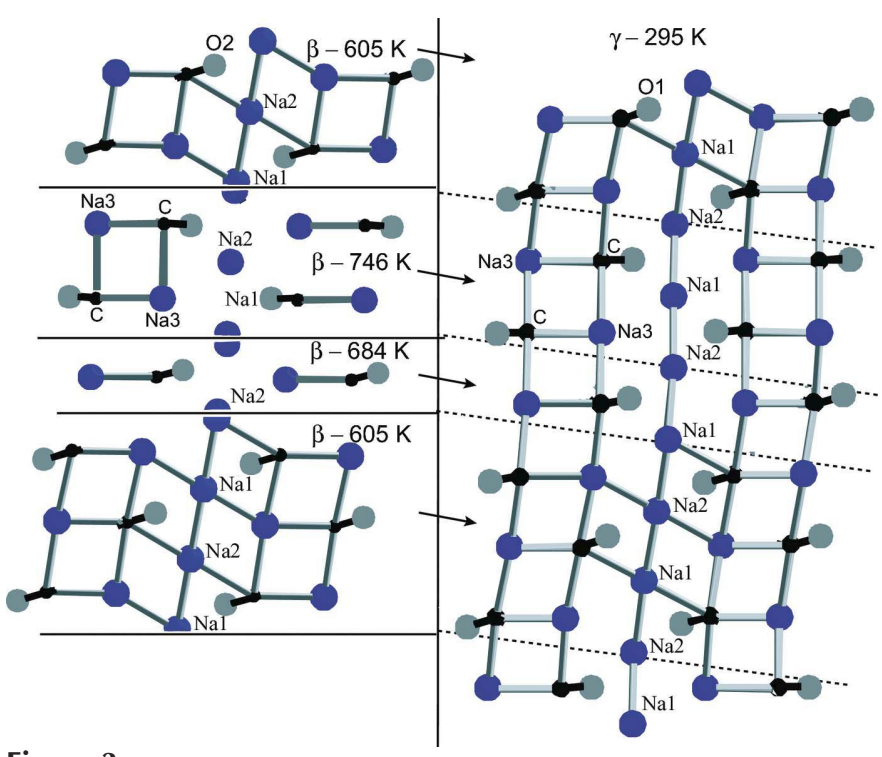

Figure 3

Analogy of the modulation wave and the temperature dependence in the $\{110\}_{\text {hex }}$ family of planes. Interatomic distances smaller than $3.1 \AA$ are shown by solid lines. The temperature-dependent evolution of the $\mathrm{C}-$ $\mathrm{Na} 2$ distance, $\mathrm{Na} 3-\mathrm{C}-\mathrm{Na} 3$ angle and $\mathrm{O} 2$ displacement in the $m_{M}$ plane in the $\beta$ phase (left). Following the modulation along $t$, the same types of magnitudes occur again for $\mathrm{C}-\mathrm{Na} 1, \mathrm{Na} 3-\mathrm{C}-\mathrm{Na} 3$ angle and $\mathrm{O} 1$ displacement in the $m_{V}$ plane along $\mathbf{c}$ in the $\gamma$ phase (right). channel atoms, the other corresponding to the interlayer $\mathrm{C}-$ $\mathrm{Na} 3$ distance. Both distances are located on a series of atomic planes parallel to $\{110\}_{\text {hex }}$ (Fig. 1). In the hexagonal $\alpha$ phase, these atomic planes correspond to the mirror planes $m$. In the monoclinic modifications, only one of the planes, namely $m_{M}$ (Fig. 1), remains as a mirror plane. In this context, the other two are called $m_{V}$ (virtual mirror) planes (Fig. 1).

Our analysis (Fig. 2) of the structural data shows that the series of phase transitions observed by decreasing temperature is associated with the formation of $\mathrm{C}-\mathrm{Na}$ bonds in the family of $\{110\}_{\text {hex }}$ atomic planes. The first step is the formation of bonds in the monoclinic $m_{M}$ mirror plane. The second step is the formation of $\mathrm{C}-\mathrm{Na}$ bonds in the two $m_{V}$ planes of the incommensurate $\gamma$ phase: two anti-phase waves of the $\mathrm{C}-\mathrm{Na}$ distances appear respectively in two $m_{V}$ planes along the internal $t$ axis. The analogy of the temperature dependence
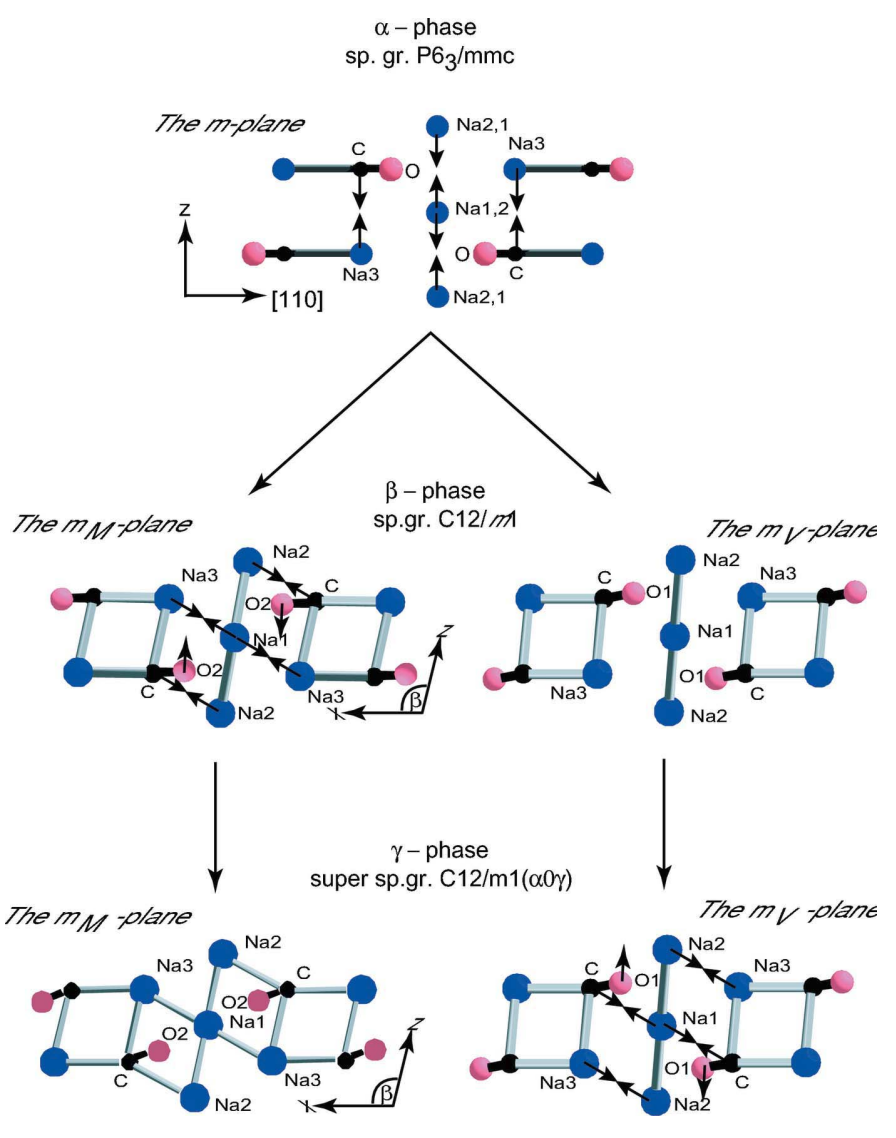

Figure 4

Phase transition scheme of $\mathrm{Na}_{2} \mathrm{CO}_{3}$. The structural transformations take place in the family of atomic planes $\{110\}_{\text {hex }}$. In the $\alpha$ phase the interlayer $\mathrm{C}-\mathrm{Na} 3$ distance decreases from 3.35 ( $972 \mathrm{~K}$ ) to $3.23 \AA$ (754 K). In the $\beta$ phase significant changes appear on the mirror $m_{M}$ plane: the decrease in the $\mathrm{C}-\mathrm{Na} 2$ distances from 3.4 to $3.18 \AA$ and $\mathrm{C}-\mathrm{Na} 3$ from 3.23 to $3.1 \AA$ induces an increase of the monoclinic $\beta$ angle from $90(754 \mathrm{~K})$ to $99.33^{\circ}$ $(605 \mathrm{~K})$; the decrease in the $\mathrm{C}-\mathrm{Na} 2$ distance is associated with a shift of O2. In the $\gamma$ phase significant transformations appear in the two $m_{V}$ planes as anti-phase modes along $t$ : the $\mathrm{C}-\mathrm{Na} 1$ distances vary between 3.4 and $3.0 \AA$; the decrease of the $\mathrm{C}-\mathrm{Na} 1$ distance is associated with a shift of O1. In the $\gamma$ phase the content of the $m_{M}$ plane is slightly affected by temperature: the monoclinic $\beta$ angle changes only by $2.5^{\circ}$ between 605 and $110 \mathrm{~K}$; the convergence of the $\mathrm{C}-\mathrm{Na} 3$ and $\mathrm{C}-\mathrm{Na} 2$ distances also induces a convergence in the $\mathrm{O} 2$ atomic position. 


\section{research papers}

and the modulation wave of the $\mathrm{C}-\mathrm{Na}$ distances in the planes of the $\{110\}_{\text {hex }}$ family is illustrated in Fig. 3. This analogy confirms the common nature of the $\mathrm{C}-\mathrm{Na}$ interactions and points to a unique mechanism, inducing the sequence of phase transitions.

The structural transformation scheme is described in Fig. 4. A few remarkable features extracted from the distancetemperature diagrams of Fig. 2 should be underlined in this context.

(i) At the $\alpha \rightarrow \beta$ transition, which occurs at $754 \mathrm{~K}$, the interlayer distance $\mathrm{C}-\mathrm{Na} 3=3.23 \AA$ splits into two distances, 3.1 and $3.35 \AA$, indicating a discontinuity in the structural transformation.

(ii) The shortest value matches the bond range and does not vary in the temperature range of the $\beta$ phase. Another value reaches the bond range close to the $\beta \rightarrow \gamma$ transition temperature of $605 \mathrm{~K}$. At this temperature, the interlayer distance splits once more, varying from 3.1 to $2.9 \AA$ along the internal $t$ axis in the $\gamma$ phase. This splitting again indicates a discontinuity of the structural transformation at $605 \mathrm{~K}$.

(iii) In the temperature range of phase $\beta$ the variation in the $\mathrm{C}-\mathrm{Na} 3$ interlayer distances is independent of the continu-

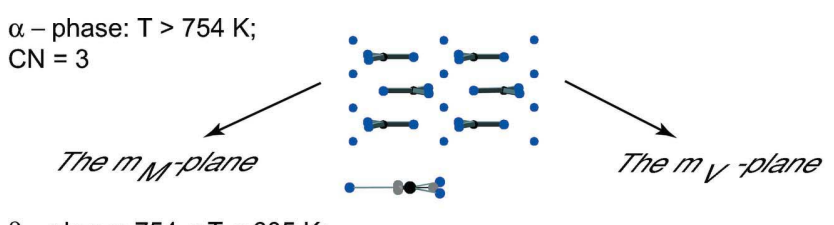

$\beta$ - phase: $754<\mathrm{T}<605 \mathrm{~K}$;

$\mathrm{CN}=4$
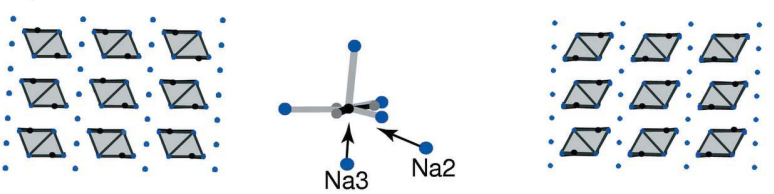

$\gamma^{\prime}-$ phase: $530<\mathrm{T}<605 \mathrm{~K}$;

$\mathrm{CN}=5$
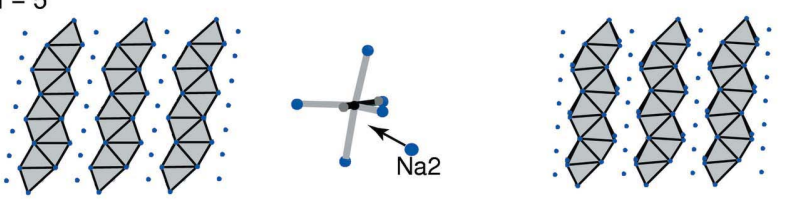

$\gamma$ - phase: $170<\mathrm{T}<530 \mathrm{~K}$ $\mathrm{CN}=6-7$

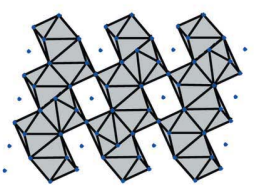

$\delta$ - phase: $T<170 \mathrm{~K}$; $\mathrm{CN}=7$

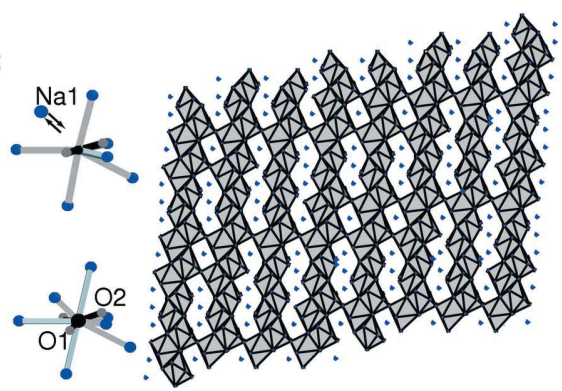

Figure 5

Evolution of the $\mathrm{Na}$ environment in the vicinity of the $\mathrm{C}$ atom. The coordination number $(\mathrm{CN})$ of the $\mathrm{C}$ atom is limited to the $\mathrm{C}-\mathrm{Na}$ distances of up to $3.1 \AA$. The projections of $\left[\mathrm{CNa}_{n}\right]$ polyhedra are plotted in two different planes of the $\{110\}_{\text {hex }}$ family. Non-bonded $\mathrm{Na}$ ions are shown by circles. The environment of the $\mathrm{C}$ atom including both $\mathrm{Na}$ ions and $\mathrm{O}$ atoms is also presented (center). ously decreasing $\mathrm{C}-\mathrm{Na} 2$ distance, which causes the intensive increase of the monoclinic angle in the $m_{M}$ plane (Fig. 4) that justifies the $\epsilon_{5}=a \cos \beta^{*} / a_{0}$ shear strain component.

(iv) This $\mathrm{C}-\mathrm{Na} 2$ distance $(3.18 \AA$ at the transition temperature of $605 \mathrm{~K}$ ) can still decrease in order to form a bond. This distance decreases continuously, reaching the bond value $3.1 \AA$ only at $\sim 530 \mathrm{~K}$ (Fig. 2). At this temperature we can expect the splitting of the $\mathrm{C}-\mathrm{Na} 1$ distance by anti-phase modulations in the $m_{V}$ atomic planes. This occurs only after the formation of all the possible $\mathrm{C}-\mathrm{Na}$ bonds in the $m_{M}$ atomic plane.

Therefore, we propose an additional phase transformation at $530 \mathrm{~K}$ : incommensurate $\gamma^{\prime}$-incommensurate $\gamma$. This is confirmed by the experimental X-ray diffraction pattern relating to the thermal treatment and the temperature dependence of the $\alpha$ component of the $\mathbf{q}$ vector, both published in de Wolff \& Tuinstra (1986); Figs. 1 and 7, respectively. The IR spectra of the $\mathrm{CO}_{3}$ group discussed below are also compatible with this assumption. The temperaturedependent modulation vector $\mathbf{q}$ becomes rational, $\mathbf{q}=$ $\frac{1}{6} a^{*}+\frac{1}{3} c^{*}$, at the $\gamma$ to $\delta$ transition. At this temperature, the commensurate ordering of the $\mathrm{C}-\mathrm{Na}$ bonds results in a multiple monoclinic unit cell. In the lock-in $\delta$ phase, the antiphase modulation of the $\mathrm{C}-\mathrm{Na} 1$ distances observed in the incommensurate phase freezes, resulting in three short $(<$ $3.14 \AA$ ) and three long (> $3.29 \AA$ ) distances at $110 \mathrm{~K}$. It can be expected that by further decreasing the temperature, the three shorter $\mathrm{C}-\mathrm{Na} 1$ distances in the $\delta$ phase will reach the shortest possible bond distances of $3.0 \pm 0.1 \AA$.

The evolution of the $\mathrm{C}-\mathrm{Na} 3$ and $\mathrm{C}-\mathrm{Na} 1,2$ distances is associated with the corresponding evolution of the $\mathrm{Na} 1-\mathrm{Na} 2$ and $\mathrm{Na3}-\mathrm{Na} 1,2$ distances, as seen in Figs. 3 and 4. However, in contrast to the $\mathrm{C}-\mathrm{Na}$ distances, the $\mathrm{Na}-\mathrm{Na}$ distances decrease smoothly from 3.4 to $3.0 \AA$ and do not show any specific characteristic of a phase transition.

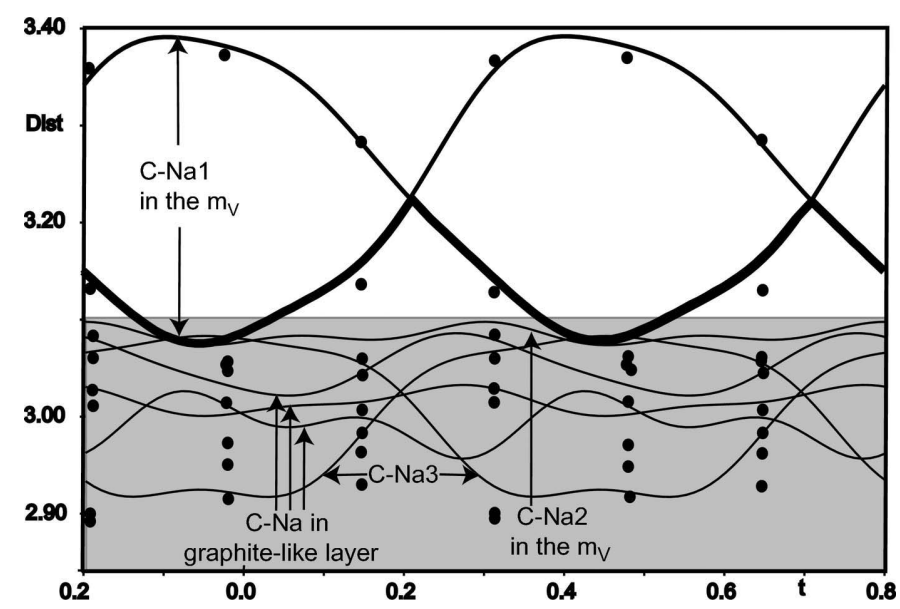

Figure 6

$t$-dependence of the $\mathrm{C}-\mathrm{Na}$ distances in the vicinity of the $\mathrm{C}$ atom in the $\gamma$ (lines) and $\delta$ (dots) phases. Six $\mathrm{Na}$ ions are always present in the second coordination sphere of $\mathrm{C}$ within the $3.1 \AA$ radius (gray area). The bold line indicates the modulation of the seventh $\mathrm{Na}$ ion in the vicinity of $\mathrm{C}$ in the $\gamma$ phase. 


\section{The Na environment of the $\mathrm{C}$ atom and the phase transition}

The formation of the $\mathrm{C}-\mathrm{Na}$ bonds which gradually appear on decreasing the temperature are associated with an increase in the number of $\mathrm{Na}$ ions in the vicinity of the $\mathrm{C}$ atoms, i.e. the coordination number $(\mathrm{CN})$ of $\mathrm{C}$ in the second coordination sphere. In Fig. 5 the temperature-dependent evolution of the $\mathrm{Na}$ environment of the $\mathrm{C}$ atom within the $3.1 \AA$ is projected on the $m_{M}$ and $m_{V}$ planes. Fig. 5 also indicates an increase in the plane density on decreasing the temperature.

It is significant that every additional bond formation (the increase of $\mathrm{CN}$ by 1 ) always relates to a new phase transition (Fig. 5, center). The three $\mathrm{Na}$ ions surrounding the $\mathrm{C}$ atom on the graphite-like layer correspond to $\mathrm{CN}=3$ in the $\alpha$ phase. At $754 \mathrm{~K}$, the $\alpha \rightarrow \beta$ transition is characterized by a change of coordination number from 3 to 4 owing to the splitting of the interlayer $\mathrm{C}-\mathrm{Na} 3$ distance. The $\beta \rightarrow \gamma^{\prime}$ transition temperature at $605 \mathrm{~K}$ increases the $\mathrm{CN}$ to 5 . At $530 \mathrm{~K}$, the $\gamma^{\prime} \rightarrow \gamma$ transition temperature, the $\mathrm{CN}$ increases to 6 owing to the formation of the $\mathrm{C}-\mathrm{Na} 2$ bonds in the $m_{M}$ atomic plane. An anti-phase modulation of two $\mathrm{C}-\mathrm{Na} 1$ distances located on two $m_{V}$ atomic planes appears at this temperature, thus producing domains of $\mathrm{CN}=6$ and a smaller fraction with $\mathrm{CN}=$ 7 in the incommensurate $\gamma$ phase. The change in $\mathrm{CN}$ along the internal $t$-axis can be extracted from Fig. 6. At the $\gamma \rightarrow \delta$ transition temperature, $170 \mathrm{~K}$, the periodic sequence of $\mathrm{CN}(6$, $6,7)$ is realised once the modulation vector $\mathbf{q}$ reaches $[1 / 6,0$, 1/3] (Fig. 6). A complete stabilization of $\mathrm{CN}=7$ in the $\delta$ phase can only be reached at lower temperature.

From this description, it can be concluded that in $\mathrm{Na}_{2} \mathrm{CO}_{3}$ and by deceasing the temperature, the phase transitions are

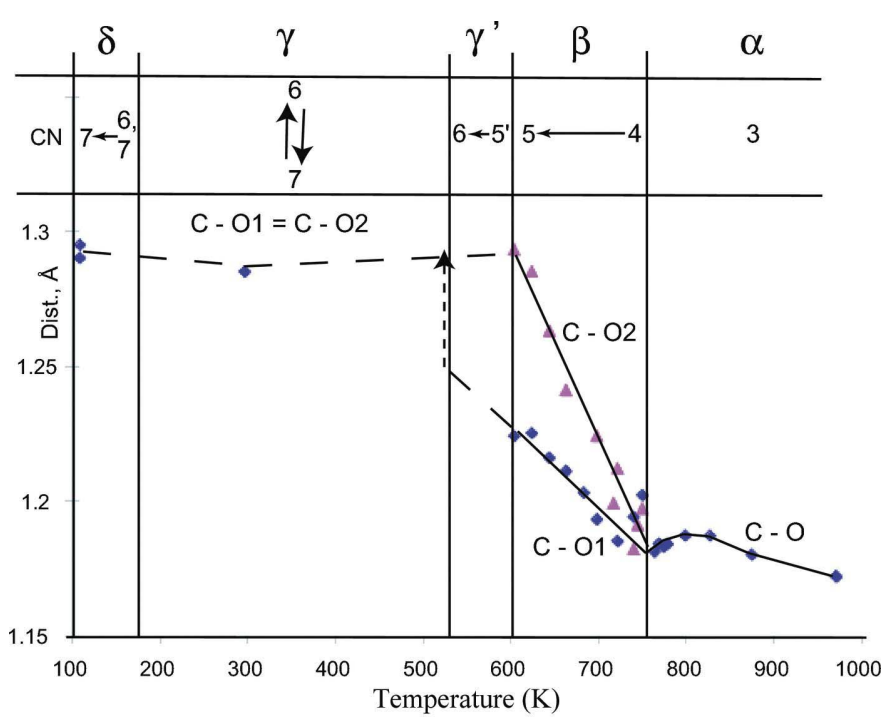

Figure 7

Correlation between the temperature dependences of the $\mathrm{C}-\mathrm{O}$ distances (dots and lines) and the numbers of $\mathrm{Na}$ ions $(\mathrm{CN})$ in the second coordination sphere $(R=3.1 \AA)$ of the $\mathrm{C}$ atom. Dashed lines are approximations. The $\mathrm{C}-\mathrm{O} 2$ and $\mathrm{C}-\mathrm{O} 1$ distances are located on the $m_{M}$ respectively $m_{V}$ planes. The distances are given without a libration correction. characterized by an increase of the number of $\mathrm{C}-\mathrm{Na}$ bonds (respectively $\mathrm{CN}$ ) in the second coordination sphere of the $\mathrm{C}$ atoms. Knowing that the intralayer bonds are constant over the whole temperature range, this number increases owing to the strengthening of both interlayer $\mathrm{C}-\mathrm{Na} 3$ interactions and interactions between the layers and the channel atoms, Na1 and $\mathrm{Na} 2$. None of the channel atoms are bonded in the $\alpha$ phase, whereas they are completely bonded to the $\mathrm{C}$ atom in the lowest $\delta$ phase.

Taking into account the crystallographic analogy between $\mathrm{C}$ and Na3 (Fig. 1), we can state that the phase transitions are linked to an increase in the non-O atom concentration close to the intersections of the most densely packed atomic planes, namely the graphite-like layers [the (001) planes] and the three planes of the $\{110\}_{\text {hex }}$ family. Both $\mathrm{C}$ and Na3 are located at these intersections. At each intersection and within $3.1 \AA$, the non-O-atom concentration increases on decreasing the temperature: in the graphite-like layer of the $\alpha$ phase (754 $972 \mathrm{~K}$ ) only one atom can be found within this radius. In the $\beta$ phase $(605-754 \mathrm{~K})$ the number increases to two owing to the interlayer contacts. The concentration increases from 2 to 2.5

$\beta$-phase from 605 to $746 K$

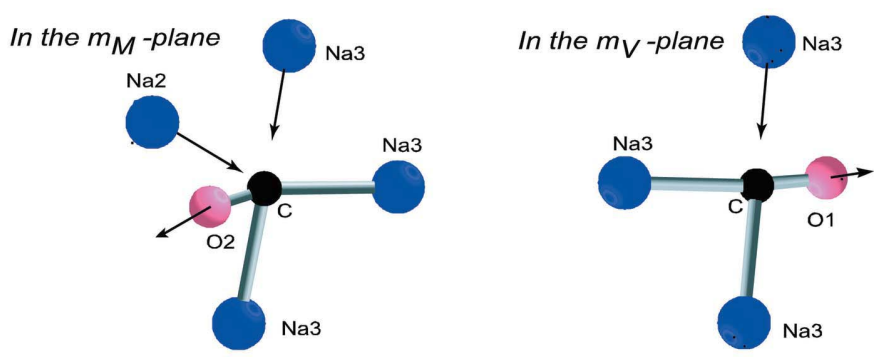

Na2-C: $3.45 \rightarrow 3.18 A$ C-O2: $1.19 \rightarrow 1.29 \AA$

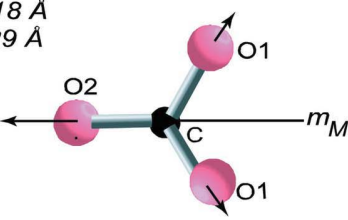

Na3-C: $3.30 \rightarrow 3.10 \AA$ C-O1: $1.17 \rightarrow 1.22 \AA$

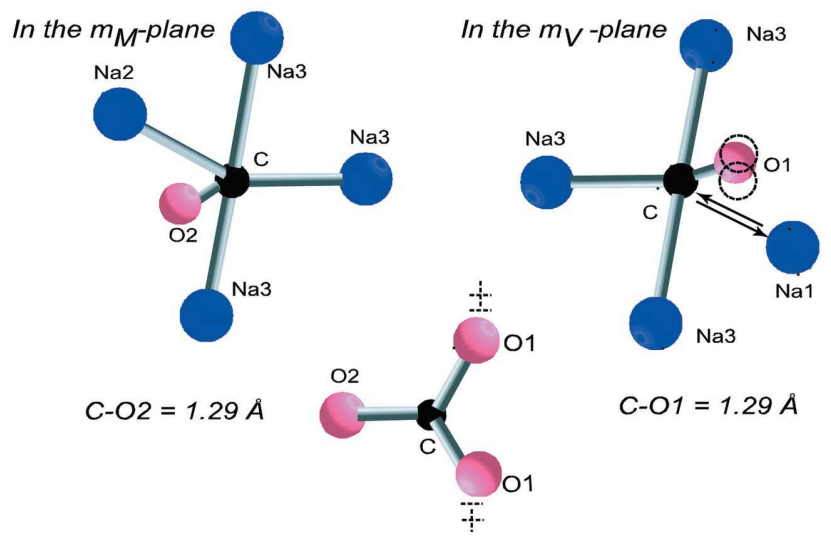

Figure 8

Correlation between the first ( $\mathrm{O}$ atoms) and the second ( $\mathrm{Na}$ ions) coordination spheres of $\mathrm{C}$ in the $\beta$ and $\gamma$ phases. 
atoms, which corresponds to the progressive deformation of the atomic arrangement on the $m_{M}$ plane of the $\gamma^{\prime}$ phase (530$605 \mathrm{~K}$ ). A modulation of the concentration from 2.5 to 3 atoms appears in the $\gamma$ phase owing to the atomic arrangement on the $m_{V}$ planes $(170-530 \mathrm{~K})$. At $110 \mathrm{~K}$, the $\delta$ phase, we find an ordered sequence of atom concentration $(2.5,2.5,3)$ aligned along q. We postulate a value of 3 for the atom concentration as a limiting value at the lowest temperature of the $\delta$ phase.

\section{Influence of the $\mathrm{Na}$ environment on the $\mathrm{CO}_{3}$ group}

The temperature dependence of the $\mathrm{Na}$ environment of the $\mathrm{C}$ atom is correlated to both a tilt of the $\mathrm{CO}_{3}$ group (Fig. 5) and the $\mathrm{C}-\mathrm{O}$ distances (Fig. 7). This correlation is illustrated in Fig. 8. The $\mathrm{O}$ atoms attempt to avoid the screening for the $\mathrm{C}-$ $\mathrm{Na}$ interactions. This is why the $\mathrm{O}$ atom deviates gradually from the $\mathrm{C}-\mathrm{Na}$ line in the corresponding plane (Fig. 3-5 and 8). As a consequence, we observe a tilt of the $\mathrm{CO}_{3}$ triangle. As can be deduced from Fig. 8, the increase of the $\mathrm{C}-\mathrm{Na}$ bond number and the associated decrease of the bond length induces an increase of the $\mathrm{C}-\mathrm{O}$ distances in the family of atomic planes $\{110\}_{\text {hex }}$ (Fig. 7). Therefore, the difference between the $\mathrm{C}-\mathrm{O} 1$ (in $m_{V}$ ) and $\mathrm{C}-\mathrm{O} 2\left(\right.$ in $m_{M}$ ) distances, the very large anisotropy of their thermal libration (discussed by Wolff $\&$ Tuinstra, 1986, for $\alpha$ and $\beta$ phases), and the difference in the $t$-dependent displacements between $\mathrm{O} 1$ and $\mathrm{O} 2$ revealed by Dušek et al. (2003) are a direct consequence of the lengths and numbers of $\mathrm{C}-\mathrm{Na}$ bonds. In other words, the carbonate group is not a fully rigid unit. Moreover, the

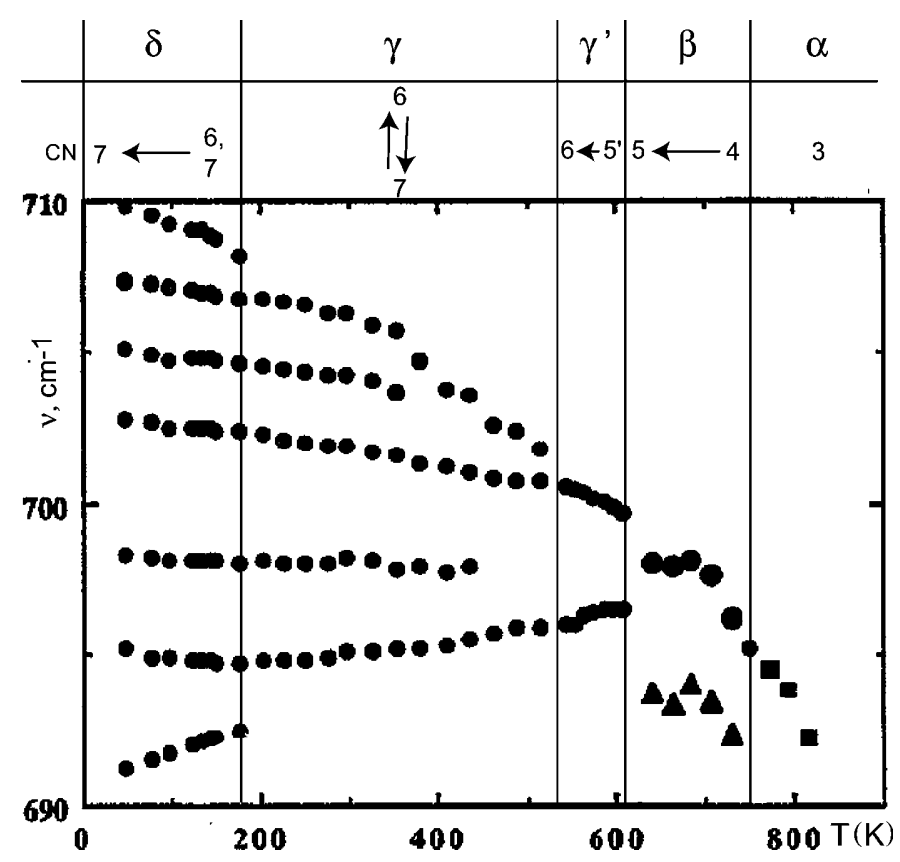

Figure 9

Correlation between the IR spectra of the $\mathrm{CO}_{3}$ internal modes (after Harris \& Salje, 1992) and the Na environment of the $\mathrm{C}$ atom at different temperatures. The solid vertical lines correspond to the phase-transition temperatures. complex IR absorption spectra of the internal modes of the carbonate ion revealed in Harris \& Salje (1992) are also directly correlated with the $\mathrm{CN}$ of the $\mathrm{C}$ atom (Fig. 5 and 9).

\section{Conclusions}

We have established a direct link between the $\mathrm{C}-\mathrm{Na}$ distances and the sequence of phase transformations in $\mathrm{Na}_{2} \mathrm{CO}_{3}$, which is systematic over the full range of phases. By decreasing the temperature, the consecutive increase of the $\mathrm{C}-\mathrm{Na}$ bond number $(\mathrm{CN})$ in the second coordination sphere of the $\mathrm{C}$ atom as $3 \rightarrow 4 \rightarrow 5 \rightarrow 6-7 \rightarrow 7$ fits respectively the $\alpha \rightarrow \beta \rightarrow \gamma^{\prime}$ (predicted) $\rightarrow \gamma \rightarrow \delta$ phase transitions. This is the main conclusion of the present work. Consequently, we propose to directly associate the $\mathrm{C}-\mathrm{Na}$ bond formation with the phase transition mechanism:

(i) The $\alpha \rightarrow \beta$ phase transition which appears at $754 \mathrm{~K}$ is characterized by a splitting of the $\mathrm{C}-\mathrm{Na} 3=3.23 \AA$ interlayer distance ( $\alpha$ phase) into 3.1 and $3.35 \AA$ ( $\beta$ phase $)$. This splitting clearly defines the internal structure discontinuity, but does not affect the lattice parameters and, therefore, cannot be characterized by or be caused by any spontaneous shear strain, including $\epsilon_{5}$ discussed by Swainson et al. (1995) for $\mathrm{Na}_{2} \mathrm{CO}_{3}$ as the primary order parameter of the second-order ferroelastic $\alpha \rightarrow \beta$ phase transition. However, this interlayer $\mathrm{C}-\mathrm{Na} 3$ bond formation can be a reason for the widely discussed 'lattice melting' observed at the phase transition (Swainson et al., 1995; Harris \& Dove, 1995, Harris et al., 1996). Therefore, the $\alpha \rightarrow \beta$ phase transition needs a more consistent interpretation.

(ii) The $\beta \rightarrow \gamma^{\prime}$ transformation is a first-order phase transition of the commensurate-incommensurate type at $605 \mathrm{~K}$. The appearance of modulations affects the interlayer $\mathrm{C}-\mathrm{Na} 3$ distances; no significant modulation of the $\mathrm{C}-\mathrm{Na} 1$ distances can be observed.

(iii) We propose a new incommensurate-incommensurate phase transition, $\gamma^{\prime} \rightarrow \gamma$ at $\sim 530 \mathrm{~K}$. This transition is associated with the creation of a high-amplitude anti-phase modulation of $\mathrm{C}-\mathrm{Na} 1$ distance in two planes of the $(110)_{\text {hex }}$ family.

(iv) The $\gamma \rightarrow \delta$ 'incommensurate-lock-in' transformation is related to the ordering of the $\mathrm{C}-\mathrm{Na} 1$ bonds in three-dimensional space.

This work is supported by the Swiss National Science Foundation, grant Nos. 20-67698.02 and 200021-109470. M. Dušek is also gratefully acknowledged for his kind help.

\section{References}

Dušek, M., Chapuis, G., Meyer, M. \& Petřiček, V. (2003). Acta Cryst. B59, 337-352.

Harris, M. J. \& Dove, M. T. (1995). Modern Phys. Lett. B, 9, 6785.

Harris, M. J., Dove, M. T. \& Godfrey, K. W. (1996). J. Phys. Condens. Matter, 8, 7073-7084. 


\section{research papers}

Harris, M. J. \& Salje, K. H. (1992). J. Phys. Condens. Matter, 4, 43994408.

Hemmersbach, S., Zibrowius, B. \& Ruschewitz, U. (1999). Z. Anorg. Allg. Chem. 625, 1440-1446.

Parlinski, K. \& Chapuis, G. (1993). Phys. Rev. B, 47, 13983-13991.
Swainson, I. P., Dove, M. T. \& Harris, M. J. (1995). J. Phys. Condens. Matter, 7, 4395-4418.

Wolff, P. M. \& Tuinstra, F. (1986). Incommensurate Phases in Dielectrics, edited by R. Blinc \& A. P. Levanyuk, Vol. 2, pp. 253 281. Amsterdam: North-Holland-Elsevier. 